\title{
A THEOREM ON THE SEMIGROUP OF BINARY RELATIONS
}

\author{
JAW-CHING YANG ${ }^{1}$
}

The purpose of this note is to generalize a theorem of Zaretskii [2]. Notations and definitions used here are based on those of [1] and [2].

TheOREM. Let $X$ be an arbitrary set. The necessary and sufficient condition that the binary relation $\rho$ is a regular element of the semigroup $S_{X}$ is that $L(\rho)$ is a completely distributive complete lattice.

Proof. Necessity. Let $\rho=\rho \delta \rho$, where $\delta \in S_{X}$. Let $\sigma=\delta \rho$, then $\sigma^{2}=\sigma$ and $\rho=\rho \sigma$. It is known in [1] that $L(\sigma)$ and $L(\rho)$ are complete lattices in which joins are unions and, moreover, $L(\sigma)$ is completely distributive.

If $A \subseteq X$, then it is easy to show that $\psi(A)=\psi(\phi(A))$ and $\phi(A)$ $=\chi(\psi(A))$ where $\phi(A) \in L(\sigma), \psi(A) \in L(\rho)$ and $\chi(A) \in L(\delta)$. Define the mapping $\theta$ of $L(\sigma)$ onto $L(\rho)$ as follows: if $\phi(A) \in L(\sigma)$, then $\theta(\phi(A))=\psi(A)$. Clearly, $\theta$ preserves set-inclusion order and is one-toone. Hence, $L(\sigma)$ is completely isomorphic with $L(\rho)$. This proves that $L(\rho)$ is completely distributive.

Sufficiency. Let $L(\rho)$ be a completely distributive complete lattice. Define the binary relation $\delta$ as follows: $(x, y) \in \delta$, iff $\rho(x, y) \rho \subset \rho$. Obviously, $\rho \delta \rho \subset \rho$.

For each $z \in X$, define $K_{z}=\{\psi(\{v\}): z \in \psi(\{v\})\}$. For any $y \in X$, let $K=\left\{K_{z}: z \in \psi(\{y\})\right\}$ and $S(K)$ denote the set of mappings $s$ of $\psi(\{y\})$ into $L(\rho)$ such that for every $z \in \psi(\{y\}), s(z) \in K_{z}$. Then $\bigvee\left\{\Lambda K_{z}: z \in \psi(\{y\})\right\}=\Lambda\{\bigvee s(\psi(\{y\})): s \in S(K)\}$. Since lattice joins are unions, we have $V_{s}(\psi(\{y\})) \supseteq \psi(\{y\})$, for each $s \in S(K)$, and hence $\Lambda\left\{V_{s}(\psi(\{y\})): s \in S(K)\right\} \supseteq \psi(\{y\})$. Therefore,

$$
\bigcup\left\{\wedge K_{z}: z \in \psi(\{y\})\right\}=\bigvee\left\{\wedge K_{z}: z \in \psi(\{y\})\right\} \supseteq \psi(\{y\}) .
$$

Let $(x, y) \in \rho$. Then $x \in \psi(\{y\})$ and so there exists a $z \in \psi(\{y\})$ such that $x \in \Lambda K_{z}$. Therefore $x \in \psi(\{w\})$ for some w satisfying

$$
\psi(\{w\}) \subseteq \wedge K_{z} \subseteq \cap K_{z}=\cap\{\psi(\{v\}): z \in \psi(\{v\})\} .
$$

Received by the editors October 30, 1968.

1 The author expresses his thanks to the referee for his comments. 
But $w \delta z$ holds iff

$$
\psi(\{w\}) \subseteq \cap\{\psi(\{v\}): z \in \psi(\{v\})\} .
$$

Thus, we have $x \rho w, w \delta z$ and $z \rho y$; hence $(x, y) \in \rho \delta \rho$. It follows that $\rho \subset \rho \delta \rho$. Therefore $\rho=\rho \delta \rho$. This completes the proof.

\section{REFERENCES}

1. G. N. Raney, $A$ subdirect union representation for completely distributive complete lattices, Proc. Amer. Math. Soc. 4 (1953), 518-522.

2. K. A. Zaretskii, Regular elements of the semigroup of binary relations, Uspehi Mat. Nauk 17 (1962), 177-179. (Russian)

Institute of Mathematics, Academia Sinica, Taiwan 